\title{
False working memories? Semantic distortion in a mere 4 seconds
}

\author{
Alexandra S. Atkins and Patricia A. Reuter-Lorenz \\ University of Michigan, Ann Arbor, Michigan
}

\begin{abstract}
False memories are well-established, episodic memory phenomena: Semantically related associates are confidently and erroneously remembered as studied items. We report four experiments yielding similar effects in a working memory paradigm. Four semantically related words were retained over a brief interval. Whether or not the interval was filled with a math verification task, semantically related lures were mistakenly recognized as members of the memory set and took longer to reject than did unrelated negative probes. In a short-term recall task, semantic intrusions exceeded other errors (e.g., phonemic). Our results demonstrate false memory effects for a subspan list when a mere $4 \mathrm{sec}$ was given between study and test. Such rapid semantic errors presumably result from associative processing, may be related to familiarity-based proactive interference in working memory, and are consistent with recent models that integrate short- and long-term memory processes.
\end{abstract}

Episodic remembering is often associated with the feeling that we are, in some way, reexperiencing or reliving past events. We may vividly recall sights, sounds, tastes, and feelings associated with events that occurred minutes, days, or years ago. Despite this subjective experience, research shows that our long-term memories are not always reliable representations of the past (Roediger, 1996; Roediger \& McDermott, 1995; Schacter \& Slotnick, 2004). Such memories, rather, may differ dramatically from true events and, in some cases, may actually be "false," either in part or in their entirety, reflecting events that never truly occurred (Loftus, 1997; Roediger, 1996; Roediger \& McDermott, 1995).

Research on false memories has focused on long-term episodic memory, exploring how recall and recognition of previously studied material can become distorted, generally over a period ranging from minutes to days. In the Deese-Roediger-McDermott (DRM) paradigm (Deese, 1959; Roediger \& McDermott, 1995), participants study 15-item word lists, all of which are semantically related to an unstudied theme word. The participants then recall as many words as possible from the list or recognize studied words presented in a second list that includes studied words, the unstudied theme word, and other unassociated and unstudied foils. Investigations using the DRM paradigm consistently show that participants both falsely recall and falsely recognize unstudied theme words. Confidence ratings or remember/know judgments typically indicate moderate confidence in these decisions (e.g., Frost, 2000; Roediger \& McDermott, 1995; Schacter \& Slotnick, 2004).

Although the false memory phenomenon has been investigated largely in the long-term memory domain, re- search on short-term working memory indicates that this system too is fallible (Baddeley, 2003; Baddeley \& Hitch, 1974). Working memory is susceptible to several forms of interference, wherein accurate and timely recall or recognition can be disrupted by interfering information. The interfering effects of phonological and visual similarity were established early on (Arons \& Mason, 1999; Conrad, 1964; Logie, Della Sala, Wynn, \& Baddeley, 2000). However, despite several well-documented semantic effects, such as semantically based proactive interference (Wickens, 1973) and semantic influences on memory span (Baddeley, 1966; Crowder, 1978; see also Hulme, Maughan, \& Brown, 1991), semantic interference effects on short-term remembering were originally found to be less robust, less consistent (e.g., Baddeley, 1966, 1972; Baddeley \& Dale, 1966; Baddeley \& Levy, 1971; Dale \& Gregory, 1966; Kintsch \& Buschke, 1969; Shulman, 1970, 1972), and generally weaker than those associated with long-term memory. Consequently, semantic coding was historically considered a signature of episodic longterm memory, whereas models of verbal short-term working memory emphasized lower level phonological codes (Baddeley, 1966, 1972, 1986). This dichotomy, which is inconsistent with many current models of memory (Baddeley, 2000; Cowan, 1999, 2005; Haarmann \& Usher, 2001; Nairne, 2002), may have diverted interest away from the question of whether short-term remembering is susceptible to false memory effects.

More recently, the possibility of short-term semantic coding has become more widely recognized. Patient studies indicate that working memory may include separable components dedicated to the short-term maintenance of semantic information (Hamilton \& Martin, 2005; Martin, 
Shelton, \& Yafee, 1994). Moreover, the idea of short-term semantic representations is readily handled by models that conceptualize working memory as the activated portion of long-term memory (Cowan, 1999, 2005; Haarmann \& Usher, 2001; Nairne, 2002; Oberauer, 2002; cf. Baddeley, 2000). The view that working memory and episodic longterm memory are mediated by overlapping and shared components is also supported by evidence gleaned from brainimaging studies (e.g., Cabeza, Dolcos, Graham, \& Nyberg, 2002; Karlsgodt, Shirinyan, van Ep, Cohen, \& Cannon, 2005; Ranganath, Johnson, \& D'Esposito, 2003).

These developments are consistent with the possibility that false memories may occur rapidly, on the time scale of working memory tasks, and may not require the use of supraspan lists of memoranda. Rapidly occurring associative processes constitute the basis for one of the popular explanations of the false memory effect in long-term remembering (Roediger, McDermott, \& Robinson, 1998). According to this account, theme words semantically related to studied items become activated via associative processes in semantic memory (Collins \& Loftus, 1975; Underwood, 1965; see also Arndt \& Reder, 2003), much like semantic priming effects that are evident within seconds following exposure to a prime (Meyer \& Schvaneveldt, 1971).

Our present purpose, therefore, was to test the hypothesis that false recognition and false recall can occur within a canonical working memory paradigm, with subspan lists of memoranda. The first pair of experiments (1A and $1 \mathrm{~B})$ used recognition and recall, respectively. Memory sets of four semantically related words were followed by a 3- to 4-sec retention interval that was filled with a mathematical distractor task, making trials similar in structure to the operation span task that is widely used to investigate working memory (Smith et al., 2001; Turner \& Engle, 1989). In the recognition task (Experiment 1A), the retention interval was followed by one of three probe types: a positive probe that had appeared in the memory set for that trial, a negative probe that had not appeared and was not associated with items in the memory set, or a lure probe that was semantically associated but not part of the memory set. In the recall task (Experiment 1B), participants engaged in free recall of memory items following the retention interval. Identical procedures were used in the second pair of experiments ( $2 \mathrm{~A}$ and $2 \mathrm{~B}$ ), except that the distractor task was omitted, thereby permitting subvocal rehearsal. In all four experiments, false memory effects were robust, indicating that semantic memory distortions can occur when attempting to retain only four items over a mere 3- to 4-sec delay.

We also predicted that response time (RT) measures of recognition performance would be sensitive to the semantic association between the probe and the memory set, consistent with previous findings by Bartha, Martin, and Jensen (1998). In an item recognition task in which four unassociated words were used as memoranda, Bartha et al. found significant slowing when participants correctly rejected nonstudied synonyms or associates of the memory set. In the present experiments, we found that participants took longer to reject lures than negative probes, revealing robust semantic interference effects.

\section{EXPERIMENT 1A}

\section{Method}

\section{Participants}

The participants were 24 right-handed University of Michigan students ( 12 of them male; mean age $=19.25$ years). The participants in this experiment and in all the subsequent experiments participated for course credit.

\section{Materials}

The stimuli were presented on Dell personal computers using E-Prime 1.1 software (www.pstnet.com). Buttonpress responses were collected using a Cedrus Model RB-730 response pad (www.cedrus .com). All the stimuli appeared in a black Arial 20-point font.

Item recognition task. Forty-eight lists were created from a subset of 55 previously published lists shown to elicit episodic false memories (Roediger, Watson, McDermott, \& Gallo, 2001). Each list consisted of four semantically related words, all associates of a common theme word. Only one memory set of four words was created from each original list. These 48 new lists were divided into four groups of 12 four-word lists (Groups A-D) that were equated in mean backward associative strength $(M=.34$ for each group of lists). Theme words served as the probes on all the trials, and no theme words were repeated for a given participant. Each participant completed 36 trials: 12 trials were paired with a lure, which was the theme word associated with that list; 12 trials were paired with an unrelated negative probe, which was a theme word associated with a nonpresented list; and 12 trials were paired with a positive probe. On positive probe trials, one item from the constructed list was replaced with the theme word for that list, which also served as the positive probe. Probe type was counterbalanced with word lists across participants, so that for one quarter of all the participants, lists in Group A were paired with lure probes, lists in Group B with negative probes, and lists in Group $\mathrm{C}$ with positive probes. Themes associated with Group D lists served as the unrelated negative probes. There were a total of four counterbalanced groups, with $N / 4$ subjects in each. This procedure ensured that all the participants encountered the same probes, all theme words, but in different contexts, as lures, negative probes, or positive probes. Trials were presented in random order for each participant. No participant was exposed to a given theme or probe more than once during the experiment.

Distractor task. A math equation verification task based on the operation span task (Turner \& Engle, 1989) was adapted from Smith et al. (2001). During the retention interval of the memory task, the participants viewed a completed math equation, decided whether or not it was solved correctly, and responded accordingly. In each equation, the first operation was always multiplication or division; the second was always addition or subtraction.

\section{Procedure}

The participants first provided written informed consent; all research was approved by the Behavioral Sciences Institutional Review Board at the University of Michigan. A short set of practice trials, with memory sets consisting of four unrelated words, then familiarized them with the task demands. At the beginning of each trial, the participants saw a black fixation cross, which turned white $500 \mathrm{msec}$ prior to the presentation of the memory set. The four memory set items, printed in lowercase font, then appeared for $1,200 \mathrm{msec}$. The retention interval varied randomly between 3,000 and 4,000 msec, during which the participants completed the math verification distractor task. The math equation was displayed for $3,000 \mathrm{msec}$, appearing randomly $0,150,250$, or $500 \mathrm{msec}$ following the offset of the memory set. The participants made a left-handed buttonpress response to indicate whether the math equation was solved correctly or incorrectly. A fixation cross appeared during the portion of the retention interval not occupied by the math equation $(0,150,250$, or $500 \mathrm{msec})$. Next, the probe word appeared in capital letters at the center of the screen. Using their right hand, the participants pressed one button to indicate that the probe word had 
appeared in the memory set, disregarding case, and another button if it had not. A black fixation cross appeared during the intertrial interval, which varied randomly between 1,500 and 2,000 msec. The participants were instructed to respond as accurately as possible on the math verification task and to respond as quickly and accurately as possible on the item recognition task.

\section{Results and Discussion}

Mean accuracy on the math task was $0.81(S E=.02)$. Mean probe accuracy and RTs for each probe type were compared using paired $t$ tests. Significant values are reported after Bonferroni correction for multiple comparisons, unless otherwise noted. Effect sizes for correlated designs were computed using original standard deviations for each condition mean (Dunlop, Cortina, Vaslow, \& Burke, 1996).

Our primary comparisons of interest were the accuracy and RT differences between negative probes and lures. Table 1 shows the proportion of yes responses for each probe type. The participants were nearly twice as likely to falsely recognize lure probes than negative probes as members of the memory set, and this difference was significant $[t(23)=3.43, p<.01, d=.82]$. The participants were more accurate in responding to positive (correct yes responses) versus lure probes (correct $n o$ responses) $[t(23)=$ $2.34, p<.05, d=.58]$. There was no accuracy difference between responses to positive and negative probes $[t(23)=$ 0.98 , n.s.]. Mean correct RTs for negative, lure, and positive probe trials are displayed in Figure 1A. RTs for lure probes were significantly longer than those for both negative probes $[t(23)=5.04, p<.001, d=.70]$ and positive probes $[t(23)=4.17, p<.001, d=.75]$. No reliable differences were observed between RTs to positive and negative nonrelated probes $[t(23)=0.30$, n.s. $]$, indicating that the participants were equally fast in correctly rejecting negative probes and correctly recognizing positive probes.

In this experiment, the three probe types occurred equally often. Consequently, completely accurate performance required negative responses on two thirds of the trials, which could lower the accuracy for positive probes. To reduce this no bias and to ensure that any such bias did

Table 1

Mean Proportion of Positive, Negative, and Lure Probes to Which Participants Responded Yes

\begin{tabular}{|c|c|c|c|}
\hline & \multirow{2}{*}{$\begin{array}{l}\text { Probe } \\
\text { Type }\end{array}$} & \multicolumn{2}{|c|}{$\begin{array}{l}\text { Proportion of } \\
\text { Yes Responses }\end{array}$} \\
\hline & & $M$ & $S E$ \\
\hline \multirow[t]{3}{*}{ Experiment 1A: Distraction } & Positive & .80 & .03 \\
\hline & Negative & .16 & .03 \\
\hline & Lure & .31 & .04 \\
\hline \multirow[t]{3}{*}{ Experiment 2A: No Distraction } & Positive & .96 & .01 \\
\hline & Negative & .01 & .01 \\
\hline & Lure & .09 & .03 \\
\hline
\end{tabular}

Note-A yes response indicates that the probe was recognized as a member of the memory set. Positive probes are those that did appear in the memory set. Negative probes were not members of the memory set, nor were they semantically related to memorized items. Lure probes were not present in the memory set but were semantically related to items in that set. In both experiments, lures were falsely recognized significantly more often than negative probes.
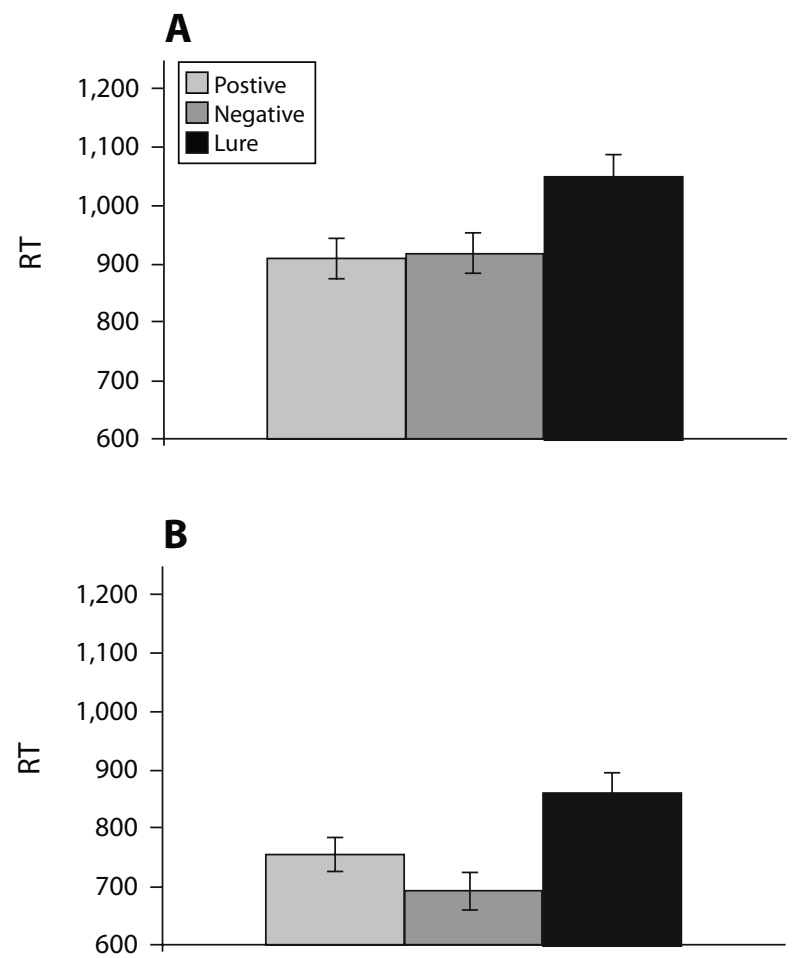

Figure 1. Mean response times (RTs, in milliseconds) for correct responses to positive, negative, and lure probes in Experiment $1 \mathrm{~A}$, distraction (A) and Experiment $2 \mathrm{~A}$, no distraction (B). The semantic interference effects, defined as the RT difference for correct rejections of lures versus unrelated negative probes, were 134 and 166 msec for Experiments $1 \mathrm{~A}$ and 2A, respectively.

not contribute to our false recognition effects, 18 new participants performed a similar experiment in which there were equal numbers of yes and no trials. Positive probe recognition was higher, and accuracy and RT differences between lures and negative probes remained highly significant. ${ }^{1}$ The subsequent recognition experiment $(2 \mathrm{~A})$ used an equal distribution of probe types because this design is more efficient for examining false recognition errors.

\section{EXPERIMENT 1B}

False recognition of semantically related lures is well documented in episodic memory. Experiment 1A demonstrated that this effect can occur on the timescale of a working memory task. However, in the long-term memory domain, false memories also manifest as semantic intrusions in free recall. The following experiment demonstrated false recall in short-term remembering when there is distraction present in the retention interval.

\section{Method}

\section{Participants}

The participants were 18 new right-handed University of Michigan students ( 9 of them male; mean age $=19.83$ years).

\section{Materials and Procedure}

This experiment used the same 48 four-item lists as those utilized in Experiment 1A, except that theme words were never presented 
as memoranda. No theme or memorandum was ever repeated. Each memory set appeared for $1,200 \mathrm{msec}$, followed by a 3- to 4-sec retention interval during which the participants completed the math distractor task described above. Next, a prompt appeared instructing the participants to say the words from the memory set aloud, in any order. Vocal responses were recorded directly onto the computer hard drive with the use of a standard Labtec microphone and HarddiskOgg audio capture software (Copyright 2004 Jan Lellmann/ Fridgesoft). The recall period was untimed.

\section{Results and Discussion}

Recall responses were transcribed and coded manually as either correct or incorrect. Incorrect responses were further classified as follows: (1) semantic - that is, the word was the theme word for the trial, an associate of that theme (not presented, but listed on the original 15-item DRM list from which our 4-item list was created; Roediger et al., 2001), or a word judged by two trained coders as being related in meaning to two or more items in the memory set; (2) phonological (the word sounded like 1 or more items in the memory set and was not a semantic associate of the theme word); or (3) other, a category that included both repeated (correct or incorrect) responses and recalled words that were related in neither meaning nor sound to those in the memory set. Note that semantic errors were classified conservatively, in that words associated with only one member of a memory set and words that did not meet the criteria for any category (approximately $2 \%$ of the errors) were omitted from analysis. Nonword and unintelligible utterances (approximately $4 \%$ of the errors) were also omitted.

The participants correctly recalled an average of 3.22 words per trial $(S E=0.10)$. Mean recall errors by error type are displayed in Figure 2A. The main effect for error type was significant $\left[F(2,34)=15.33, p<.001, \eta^{2}=\right.$ 47]. The participants made more semantic than phonological recall errors $[t(17)=4.56, p<.01, d=1.36]$. In fact, the participants made more than twice as many semantic errors as all other error types combined, and this difference was reliable $[t(17)=3.37, p<.01, d=0.77]$. Of all the responses coded as semantic errors, $66 \%$ consisted of the theme word associated with the memory set, and $18 \%$ were nonpresented (semantically associated) items from the original DRM lists. Mean recall positions for semantic, phonological, and other errors were 3.01 ( $S E=$ $0.14), 2.46(S E=0.24)$, and $2.75(S E=0.27)$, respectively, which were not significantly different. Mean math accuracy was $.81(S E=.02)$.

\section{EXPERIMENT 2A}

Experiments $1 \mathrm{~A}$ and $1 \mathrm{~B}$ demonstrated that false recognition and false recall errors can occur with a four-item memory set retained over a 3 - to 4 -sec interval. Furthermore, lure probes that were correctly rejected produced longer RTs than did correct rejections of negative unrelated probes, consistent with the possibility of semantic interference.

These rapid semantic distortions were evident in a working memory task when the retention interval was filled with distraction. The completion of a math verification task while words were held in memory is also required in the operation span task (Turner \& Engle, 1989), which is widely used as a measure of working memory capacity (see, e.g., Reuter-Lorenz \& Jonides, 2007). The use of this sort of distractor task during short-term retention, therefore, has a strong precedence in the working memory literature (Miyake \& Shah, 1999).

However, the math verification requirement presumably interfered with subvocal rehearsal of list items and may have encouraged the use of episodic memory processes. If true, then omitting the distractor task should permit verbatim retention of four items for $4 \mathrm{sec}$ and possibly eliminate the disproportional incidence of false memory errors. In Experiments $2 \mathrm{~A}$ and 2B, we examined the extent to which false recognition and recall, respectively, would occur when the math verification task was omitted. If false memories persisted in the absence of distraction, these errors could not be simply attributed to the disruption of rote rehearsal.
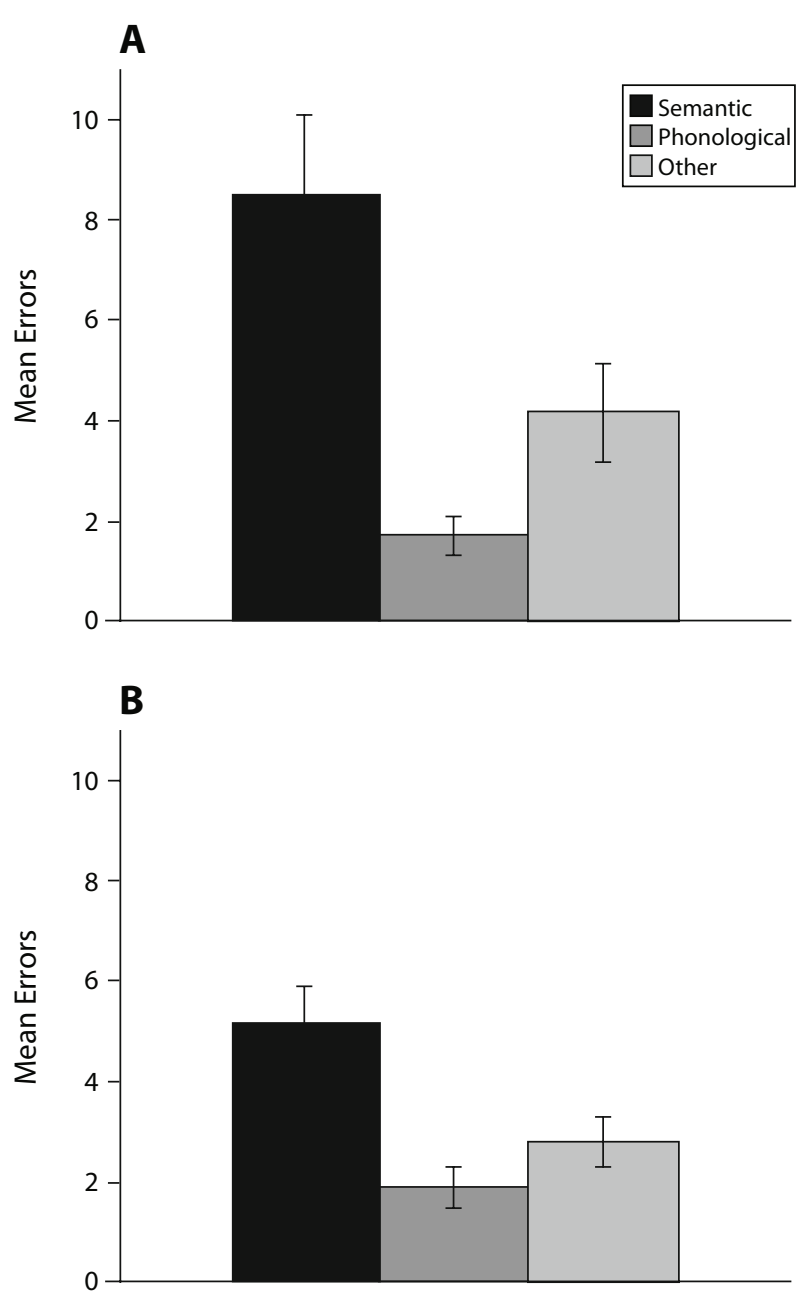

Figure 2. Mean number of semantic, phonological, and other recall errors in Experiment 1B, distraction (A) and Experiment 2B, no distraction (B). See the text for details. 


\section{Method}

\section{Participants}

Twenty-five new right-handed University of Michigan students (12 of them male; mean age $=20.3$ years) participated.

\section{Materials and Procedure}

The experiment was the same as Experiment 1A, except that the math verification task was omitted. To better equate the perceptual and response demands of the two experiments, a row of empty boxes appeared in place of the math problem during the retention interval; the participants were required to make a left-handed buttonpress response to the onset of the boxes.

\section{Results and Discussion}

Mean proportions of yes responses for each probe type are shown in Table 1. As in Experiment 1A, there was a significant false memory effect, in that the participants were more likely to falsely recognize lure probes than negative probes as members of the memory set $[t(24)=$ $3.13, p<.01, d=0.79]$. Accuracy for positive and negative probes did not differ $[t(24)=2.24$, n.s. $]$.

Mean correct RTs for negative, lure, and positive probe trials are displayed in Figure 1B. As in Experiment 1A, correct responses to lure probes took significantly longer than responses to negative probes $[t(24)=7.0, p<.001$, $d=1.04]$ and positive probes $[t(24)=3.76, p<.01, d=$ $0.68]$. Once again, the RT difference between negative and positive probes was not reliable $[t(24)=2.40$, n.s.].

In order to further examine the effects of distraction, $2 \times 2$ mixed participants ANOVAs were performed to compare accuracy and RT (correct trials) for negative and lure probes with and without distraction. The results showed main effects of distraction on both probe accuracy $\left[F(1,47)=29.91, p<.001, \eta^{2}=.39\right]$ and $\mathrm{RT}[F(1,47)=$ $\left.19.73, p<.001, \eta^{2}=.30\right]$, indicating that the presence of distraction reliably decreased accuracy and lengthened RT. There were no interactions between probe type and distraction on either dependent measure, indicating that the false recognition and semantic interference effects did not reliably differ as a function of distraction.

\section{EXPERIMENT 2B}

\section{Method}

\section{Participants}

The participants were 20 new right-handed University of Michigan students ( 11 of them male; mean age $=18.75$ years).

\section{Materials and Procedure}

The experiment was the same as Experiment 1B, except that the math verification task was omitted and replaced with the simple keypress to the onset of boxes, as in Experiment 2A.

\section{Results and Discussion}

Recall responses were transcribed and coded manually as either correct or incorrect. Incorrect responses were further classified as semantic, phonological, or other, as described in Experiment 1B. The error-coding criteria were the same as those used in Experiment 1B. Six percent of the errors that did not meet the criteria for any error category and another $6 \%$ that were non- words and unintelligible utterances were omitted from the analysis.

The participants correctly recalled an average of 3.51 words per trial $(S E=0.06)$. Mean recall errors by error type are displayed in Figure 2B. The main effect for error type was significant $\left[F(2,38)=11.27, p<.001, \eta^{2}=.37\right]$. The participants made more than twice as many semantic as phonological recall errors $[t(19)=3.89, p<.01, d=$ 1.26]. As in Experiment 1B, the participants made more semantic errors than all other error types combined, and this difference was reliable $[t(19)=3.01, p<.05, d=$ $0.86]$. Of all the responses coded as semantic errors, $46 \%$ consisted of the theme word associated with the memory set, and 24\% were nonpresented items from the original DRM lists (Roediger et al., 2001). Mean recall positions for semantic, phonological, and other errors were 2.93 $(S E=0.16), 2.34(S E=0.23)$, and $2.76(S E=0.21)$, respectively, and these did not differ reliably.

A post hoc independent samples $t$ test compared recall accuracy with and without distraction. As was expected, recall accuracy was higher when no distraction was presented during the retention interval $[t=2.56, p<.05, d=$ $0.82]$. In order to further examine the effects of distraction, a $2 \times 2$ mixed participants ANOVA compared the rate of recall errors (semantic, phonological, and other) with and without distraction. The results showed a main effect of error type $\left[F(2,72)=26.95, p<.01, \eta^{2}=.43\right]$, reflecting the prevalence of semantic errors, and a marginal error type $\times$ distraction interaction $[F(2,72)=3.11, p=.051$, $\left.\eta^{2}=.08\right]$. Examination of the means (Figure 2B) suggests a trend toward an increase in semantic and other errors in the presence of distraction and a relatively stable rate of phonological errors.

\section{GENERAL DISCUSSION}

In the present research, we investigated the occurrence of false memory effects in the working memory domain and demonstrated two robust phenomena: First, semantically related lures were falsely recognized and falsely recalled a mere 3-4 sec following study. False recognition effects occurred reliably whether or not the retention interval was filled with a distracting task. The recall results from Experiment 2B are particularly striking, in that semantic intrusions were prevalent after a brief retention interval during which the participants were free to engage in subvocal rehearsal. These false recall errors indicate that shortterm memory distortions do not require the presentation of a semantically associated lure probe or the completion of a distractor task during the retention interval.

Second, salient interference is evident in the RTs for correct rejections of semantically related probes. As compared with unrelated probes, semantic associates took well over $100 \mathrm{msec}$ longer to reject, whether the retention interval was filled or unfilled.

The present research introduces a procedure for generating reliable false memory errors within the temporal and set size parameters characteristic of working memory. The use of four associated memoranda distinguishes this 
work from previous investigations in which false recognition effects were less robust. For example, in a relatively recent item recognition study, Bartha et al. (1998) used memory sets of four unassociated items. As compared with the present results, the false recognition effects in Bartha et al. were weaker (Experiment 3) and did not replicate in a second experiment (Experiment 4).

To what extent might the present results be due to strategic processes that participants adopt to contend with task demands? In recognition (Experiments $1 \mathrm{~A}$ and 2A), might participants adopt a liberal response bias to lure probes, as in Miller and Wolford's (1999; cf. Roediger \& McDermott, 1999) account of false recognition effects in the long-term memory domain? This explanation assumes that participants shift their response criteria on a trial-by-trial basis, an assumption that does not hold for all circumstances (e.g.,Wixted \& Stretch, 2000) but may be more likely with only a single recognition test per list. However, in earlier versions of our short-term recognition task (Atkins \& Reuter-Lorenz, 2005), we presented three sets of four different associates from the same original DRM lists, thus probing the same list in consecutive test blocks. The false memory effect in these versions of the task was as robust as the effects we report here. These observations cannot definitively rule out the possibility that criterion shifts contribute to the rapid false recognition effects reported here; however, they do weaken this account as the sole source of the memory illusion we observe.

A strategy-based explanation of our false recall results also deserves consideration. In particular, participants could be treating the free recall task (Experiments 1B and 2B) like a forced recall task in which they are required to generate four responses on every trial. False recall in the long-term memory domain is greater for forced than for free recall (see, e.g., McKelvie, 2001; Meade \& Roediger, 2006). Our instructions, however, did not emphasize the requirement to recall four items, nor did the participants feel compelled to do so, as is evident from their average recall of 3.50 and 3.75 items in Experiments $1 \mathrm{~A}$ and $2 \mathrm{~A}$, respectively. Nevertheless, when the participants remembered fewer than four items, their metaknowledge about the relatedness of the lists may have led them to interrogate semantic memory in search of an appropriate response. This strategy would predict that semantic errors would be most likely in the fourth position. Like other recall errors, the average output position for semantic errors was the third (2.92 and 3.01 in Experiments 1A and 2A, respectively). These considerations lead us to believe that the false memory effects we observed reveal memory's extreme vulnerability to distortion.

What mechanisms, then, might underlie the robust false memory errors that we report? Three distinct hypotheses can be entertained. Common to all of them is the assumption that long-term semantic memory is the basic source of associative processing that mediates activation of thematically related lures and associates (Roediger et al., 2001). The accounts differ with respect to whether the mechanisms that access semantic codes are (1) intrinsic to working memory, (2) intrinsic to episodic memory, or (3) shared and common to both short- and long-term remembering.
Despite controversy as to whether there exists a working memory system with independent semantic components (e.g., Baddeley, 2003; Haarmann \& Usher, 2001; Hamilton \& Martin, 2005; Shivde \& Thompson-Schill, 2004), support for such a system comes from behavioral evidence (e.g., Potter, 1993), as well as from neuropsychological and neuroimaging results (e.g., Martin et al., 1994; Shivde \& Thompson-Schill, 2004), indicating that word meanings can be retained over the short term by working memory processes that are independent from phonological and visual codes. According to this model, the semantic errors in the present paradigm would arise from similarity-based interference during the encoding, maintenance, or retrieval of semantic codes, using a working memory subsystem that is specialized for semantics. A somewhat weaker form of this hypothesis may not require an entirely separable semantic working memory subsystem. For example, the episodic buffer, as proposed by Baddeley (2000), is a slave system to working memory's central executive that is separate from long-term memory and that contains multidimensional representations of information gathered from several sources, including longterm episodic memory, as well as the phonological and visuospatial buffers.

The second hypothesis follows from the view that although semantic errors in the present paradigm occur in the context of a working memory task, deeper semantic coding is the sole province of episodic memory processes that are operating in parallel with working memory. According to this view, short- and long-term false memories differ only in time course and are mediated by identical psychological processes. Phonological representations of the memoranda are maintained in working memory; however, false memories arise from episodic memory processes that are responsible for generating semantic distortions at longer delays (i.e., associative and monitoring processes operating during encoding and retrieval; see, e.g., Roediger et al., 2001). According to this view, the false memories that we observed are a product of longterm memory contributions to working memory.

The third hypothesis is that the false memory errors that we observe arise from processes that are shared by both short- and long-term remembering (see, e.g., Nairne, 2002, for a review). The model proposed by Cowan (1999) fits best in this class of hypotheses, in that long-term memory is postulated to provide the representational basis for short-term remembering. According to this view, shortterm memory provides the activation processes that select and maintain the relevant portion of long-term memory, in a manner resembling extended priming. The unitary model proposed by Nairne (e.g., 2002), in which shortterm memory, like episodic memory, is cue based, also falls into this third type of account. According to Nairne's feature model, what is retained over the short or long term is not a set of particular items but, rather, a constellation of cues that include phonological, lexical, and semantic features. At retrieval, these cues are used to reconstruct the memoranda. Accurate memory requires distinctive cues, thereby leading to the detrimental effects of phonological, as well as semantic, similarity. 
Determining which of these hypotheses offers the best account of the false memory errors we observe will depend on future research. We note, however, that the present results demonstrate a striking parallel in the memory distortions that can affect both recognition and recall over the short or long term. This convergence is consistent with the growing body of evidence indicating overlap between short- and long-term memory mechanisms (Cabeza et al., 2002; Karlsgodt et al., 2005; Ranganath et al., 2003; see also Baddeley, 2000; Cowan, 1999). Theoretical models that may best account for our findings, therefore, are ones that emphasize an integration of short- and long-term memory processes (the third type of hypothesis outlined above).

A second theoretical question posed by the present research relates to the psychological mechanisms underlying the observed interference effect. Lures took longer to correctly reject than did negative probes. The semantic relatedness of the lure item to the memory set presumably necessitated recruitment of additional processes, thereby increasing RT. What might these additional processes be? One possibility is that semantic relatedness increased the familiarity of the lure item, making it more difficult to reject than an item that had not been recently active in working memory. This need to adjudicate between an item's heightened familiarity and its status as a member of the current memory set is very similar to explanations offered to account for familiarity-based proactive interference effects that occur across trials in short-term item recognition paradigms (Jonides, Smith, Marshuetz, Koeppe, \& Reuter-Lorenz, 1998; Monsell, 1978). The increased RTs for lures could also stem from invoking source memory to decide whether the lure was actually presented or whether it was merely thought of due to strong associations with the memory set (e.g., Johnson, Hashtroudi, \& Lindsay, 1993). Source memory and the need for increased contextual coding have also been linked to familiarity-based proactive interference in short-term item recognition tasks (Badre \& Wagner, 2005; Jonides \& Nee, 2006).

We are currently using fMRI to investigate the possibility that common prefrontal mechanisms mediate semantic and familiarity-based interference effects (Atkins, Walsh, $\&$ Reuter-Lorenz, 2006). This ongoing neuroimaging research may also prove indispensable in distinguishing among the theoretical accounts of false working memory, in that it will allow us to examine the overlap and dissociations between the neural correlates of false short- and long-term remembering.

Taken together, our findings present strong evidence that semantic distortions of memory can occur over the very short term and reveal a surprising fallibility of verbatim memory processes. In mere seconds following presentation of a small memory set, participants falsely recall and recognize semantically related lures. Because the contents of working memory figure prominently in reasoning and problem-solving abilities, rapid distortions of meaning that go unchecked could have considerable impact on the quality of our decisions and the accuracy of our solutions. From a theoretical perspective, the data we report converge with a number of recent findings demonstrating considerable overlap in the operations and mechanisms of episodic and working memory (e.g., Cabeza et al., 2002; Nairne, 2002; Ranganath et al., 2003; Wagner, 1999; see also, Karlsgodt et al., 2005). False recognition and false recall may, therefore, constitute a valuable new province in which to investigate the relationship between short- and long-term remembering.

\section{AUTHOR NOTE}

Portions of this work were presented at the Annual Meeting of the Psychonomic Society, November 2005, in Toronto. The assistance of Megan Walsh, Beth Kinney, Jessica Howard, and Laura Zahodne is gratefully acknowledged. The authors thank John Jonides and Cindy Lustig for their helpful comments on earlier versions of the manuscript. This research was supported by NIA Grant R01AG18286 (to P.A.R.-L.) and NIMH Grant F31MH079536 (to A.S.A.). The content is solely the responsibility of the authors and does not necessarily represent the official views of the National Institutes of Health. Correspondence concerning this article should be addressed to P. A. Reuter-Lorenz, Department of Psychology, University of Michigan, 530 Church Street, Ann Arbor, MI 48109 (e-mail: parl@umich.edu).

\section{REFERENCES}

ARndt, J., \& Reder, L. M. (2003). The effect of distinctive visual information on false recognition. Journal of Memory \& Language, 48, $1-15$.

Arons, S. E., \& Mason, A. (1999). Effects of visual similarity on serial report and item recognition. Quarterly Journal of Experimental Psychology, 52A, 217-240.

AtKins, A. S., \& Reuter-Lorenz, P. A. (2005). "False" working memories: Memory distortion in a mere four seconds. Abstracts of the Psychonomic Society, 10, 28.

Atkins, A. S., Walsh, M. K., \& Reuter-Lorenz, P. A. (2006). False working memory: Rapid memory distortion and its neural correlates. Journal of Cognitive Neuroscience, 18(Suppl.), 140.

BADDEley, A. D. (1966). Short-term memory for word sequences as a function of acoustic, semantic, and formal similarity. Quarterly Journal of Experimental Psychology, 18, 362-365.

BAdDeley, A. D. (1972). Retrieval rules and semantic coding in shortterm memory. Psychological Bulletin, 78, 379-385.

BAdDeley, A. D. (1986). Working memory. Oxford: Oxford University Press, Clarendon Press.

BadDeley, A. D. (2000). The episodic buffer: A new component of working memory? Trends in Cognitive Sciences, 1, 417-423.

BADDELEY, A. D. (2003). Working memory: Looking forward, looking back. Nature Reviews Neuroscience, 4, 829-839.

Baddeley, A. D., \& Dale, H. C. A. (1966). The effects of semantic similarity on retroactive interference in long and short term memory. Journal of Verbal Learning \& Verbal Behavior, 5, 417-420.

Baddeley, A. D., \& Hitch, G. J. (1974). Working memory. In G. H. Bower (Ed.), The psychology of learning and motivation (Vol. 8, pp. 47-90). New York: Academic Press.

Baddeley, A. D., \& Levy, B. A. (1971). Semantic coding and shortterm memory. Journal of Experimental Psychology, 89, 132-136.

BADre, D., \& Wagner, A. D. (2005). Frontal lobe mechanisms that resolve proactive interference. Cerebral Cortex, 15, 2003-2012.

Bartha, M. C., Martin, R. C., \& Jensen, C. R. (1998). Multiple interference effects in short-term recognition memory. American Journal of Psychology, 111, 89-118.

Cabeza, R., Dolcos, F., Graham, R., \& Nyberg, L. (2002). Similarities and differences in the neural correlates of episodic memory retrieval and working memory. NeuroImage, 16, 317-330.

Collins, A. M., \& Loftus, E. F. (1975). A spreading-activation theory of semantic processing. Psychological Review, 82, 407-428.

ConRad, R. (1964). Acoustic confusions in immediate memory. British Journal of Psychology, 55, 75-84.

Cowan, N. (1999). An embedded-processes model of working memory. 
In A. Miyake \& P. Shah (Eds.), Models of working memory: Mechanisms of active maintenance and executive control (pp. 62-101). Cambridge: Cambridge University Press.

Cowan, N. (2005). Working memory capacity. New York: Psychology Press.

Crowder, R. G. (1978). Memory for phonologically uniform lists. Journal of Verbal Learning \& Verbal Behavior, 17, 73-89.

Dale, H. C. A., \& Gregory, M. (1966). Evidence of semantic coding in short-term memory. Psychonomic Science, 5, 75-76.

DEESE, J. (1959). On the prediction of occurrence of particular verbal intrusions in immediate recall. Journal of Experimental Psychology, 58, 17-22.

Dunlop, W. P., Cortina, J. M., Vaslow, J. B., \& Burke, M. J. (1996). Meta-analysis of experiments with matched groups or repeated measures designs. Psychological Methods, 1, 170-177.

Frost, P. (2000). The quality of false memory over time: Is memory for misinformation "remembered" or "known"? Psychonomic Bulletin \& Review, 7, 531-536.

HaArmann, H., \& Usher, M. (2001). Maintenance of semantic information in capacity-limited item short-term memory. Psychonomic Bulletin \& Review, 8, 568-578.

Hamilton, A. C., \& Martin, R. C. (2005). Dissociations among tasks involving inhibition: A single-case study. Cognitive, Affective, \& Behavioral Neuroscience, 5, 1-13.

Hulme, C., Maughan, S., \& Brown, G. D. A. (1991). Memory for familiar and unfamiliar words: Evidence for a long-term memory contribution to short-term memory span. Journal of Memory \& Language, 30, 685-701.

Johnson, M. K., Hashtroudi, S., \& Lindsay, D. S. (1993). Source monitoring. Psychological Bulletin, 114, 3-28.

Jonides, J., \& NEE, D. E. (2006). Brain mechanisms of proactive interference in working memory. Neuroscience, 139, 181-193.

Jonides, J., Smith, E. E., Marshuetz, C., Koeppe, R. A., \& ReuterLORENZ, P. A. (1998). Inhibition in verbal working memory revealed by brain activation. Proceedings of the National Academy of Sciences, 95, 8410-8413.

Karlsgodt, K. H., Shirinyan, D., van Ep, T. G. M., Cohen, M. S., \& CANnON, T. D. (2005). Hippocampal activations during encoding and retrieval in a verbal working memory paradigm. NeuroImage, $\mathbf{2 5}$, 1224-1231.

Kintsch, W., \& BuschKe, H. (1969). Homophones and synonyms in short-term memory. Journal of Experimental Psychology, 80, 403-407.

Loftus, E. F. (1997). Creating false memories. Scientific American, 277, 70-75.

Logie, R. H., Della Sala, S., Wynn, V., \& Baddeley, A. D. (2000). Visual similarity effects in immediate verbal serial recall. Quarterly Journal of Experimental Psychology, 53A, 626-646.

Martin, R. C., Shelton, J. R., \& Yafee, L. S. (1994). Language processing and working memory: Evidence for separate phonological and semantic capacities. Journal of Memory \& Language, 33, 83-111.

McKelviE, S. J. (2001). Effects of free and forced retrieval instructions on false recall and recognition. Journal of General Psychology, 128, 261-278.

Meade, M. L., \& Roediger, H. L., III (2006). The effect of forced recall on illusory recollection in younger and older adults. American Journal of Psychology, 119, 433-462.

Meyer, D. E., \& SchVaneveldT, R. W. (1971). Facilitation in recognizing pairs of words: Evidence of a dependence between retrieval operations. Journal of Experimental Psychology, 90, 227-234.

Miller, M. B., \& Wolford, G. L. (1999). The role of criterion shift in false memory. Psychological Review, 106, 398-405.

MiYaKe, A., \& Shah, P. (Eds.) (1999). Models of working memory: Mechanisms of active maintenance and executive control. New York: Cambridge University Press.

Monsell, S. (1978). Recency, immediate recognition memory, and reaction-time. Cognitive Psychology, 10, 465-501.
NAIRne, J. S. (2002). Remembering over the short-term: The case against the standard model. Annual Review of Psychology, 53, 53-81.

OBERAUER, K. (2002). Access to information in working memory: Exploring the focus of attention. Journal of Experimental Psychology: Learning, Memory, \& Cognition, 28, 411-421.

Potter, M. C. (1993). Very short-term conceptual memory. Memory \& Cognition, 21, 156-161.

Ranganath, C., Johnson, M. K., \& D’Esposito, M. (2003). Prefrontal activity associated with working memory and episodic long-term memory. Neuropsychologia, 41, 378-389.

Reuter-Lorenz, P. A., \& Jonides, J. (2007). The executive is central to working memory: Insights from age, performance and task variations. In A. R. Conway, C. Jarrold, M. J. Kane, A. Miyake, \& J. N. Towse (Eds.), Variations in working memory (pp. 250-270). Oxford: Oxford University Press.

Roediger, H. L., III (1996). Memory illusions. Journal of Memory \& Language, 35, 76-100.

Roediger, H. L., III, \& McDermott, K. B. (1995). Creating false memories: Remembering words not presented in lists. Journal of Experimental Psychology: Learning, Memory, \& Cognition, 21, 803-814.

Roediger, H. L., III, \& McDermott, K. B. (1999). False alarms about false memory. Psychological Review, 106, 406-410.

Roediger, H. L., III, McDermott, K. B., \& Robinson, K. J. (1998). The role of associative processes in producing false memories. In M. Conway, S. Gathercole, \& C. Cornoldi (Eds.), Theories of memory II (pp. 187-245). Hove, U.K.: Psychology Press.

Roediger, H. L., III, Watson, J. M., McDermott, K. B., \& Gallo, D. A. (2001). Factors that determine false recall: A multiple regression analysis. Psychonomic Bulletin \& Review, 8, 385-407.

SChacter, D. L., \& Slotnick, S. D. (2004). The cognitive neuroscience of memory distortion. Neuron, 44, 149-160.

Shivde, G., \& Thompson-Schill, S. L. (2004). Dissociating semantic and phonological maintenance using fMRI. Cognitive, Affective, \& Behavioral Neuroscience, 4, 10-19.

Shulman, H. G. (1970). Encoding and retention of semantic and phonemic information in short-term memory. Journal of Verbal Learning \& Verbal Behavior, 9, 499-508.

Shulman, H. G. (1972). Semantic confusion errors in short-term memory. Journal of Verbal Learning \& Verbal Behavior, 11, 221-227.

Smith, E. E., Geva, A., Jonides, J., Miller, A., Reuter-Lorenz, P., \& Koeppe, R. A. (2001). The neural basis of task-switching in working memory: Effects of performance and aging. Proceedings of the National Academy of Sciences, 98, 2095-2100.

Turner, M. L., \& ENGLE, R. W. (1989). Is working memory capacity task dependent? Journal of Memory \& Language, 28, 127-154.

UNDERWOOD, B. J. (1965). False recognition produced by implicit verbal responses. Journal of Experimental Psychology, 70, 122-129.

WAGNER, A. D. (1999). Working memory contributions to human learning and remembering. Neuron, 22, 19-22.

WiCKENS, D. D. (1973). Some characteristics of word encoding. Memory \& Cognition, 1, 485-490.

Wixted, J. T., \& Stretch, V. (2000). The case against a criterion-shift account of false memory. Psychological Review, 107, 368-376.

\section{NOTE}

Mean recognition accuracy was $.86(S E=.02), .93(S E=.01)$, and $.77(S E=.04)$ for positive, negative, and lure probes, respectively. Mean RT for correct trials was $796.31 \mathrm{msec}(S E=30.13)$ for positive probes, $810.57 \mathrm{msec}(S E=30.02)$ for negative probes, and $982.44 \mathrm{msec}(S E=$ 40.40) for lure probes. Mean accuracy and RT differences between lures and negative probes were significant $[t(17)=4.24, p<.01, d=1.22$, and $t(17)=7.39, p<.001, d=1.14$, respectively].

(Manuscript received August 7, 2006; revision accepted for publication May 26, 2007.) 